\title{
Synergistic effect of curcumin on epigallocatechin gallate-induced anticancer action in PC3 prostate cancer cells
}

\author{
Dae-Woon Eom ${ }^{1, \#}$, Ji Hwan Lee, ${ }^{2, \#}$,Young-Joo Kim ${ }^{2}$, Gwi Seo Hwang ${ }^{3}$, Su-Nam Kim ${ }^{2}$, Jin Ho Kwak ${ }^{4}$, Gab Jin Cheon ${ }^{5}$, \\ Ki Hyun Kim ${ }^{6}$, Hyuk-Jai Jang ${ }^{4}$, Jungyeob Ham ${ }^{2}$, Ki Sung Kang ${ }^{3, *} \mathcal{E}^{*}$ Noriko Yamabe ${ }^{3, *}$ \\ ${ }^{1}$ Department of Pathology, University of Ulsan College of Medicine, Gangneung Asan Hospital, Gangneung $25440,{ }^{2}$ Natural Products \\ Research Institute, Korea Institute of Science and Technology, Gangneung 25451, ${ }^{3}$ College of Korean Medicine, Gachon University, \\ Seongnam 13120, ${ }^{4}$ Department of Surgery, University of Ulsan College of Medicine, Gangneung Asan Hospital, Gangneung 25440, \\ ${ }^{5}$ Department of Internal Medicine, University of Ulsan College of Medicine, Gangneung Asan Hospital, Gangneung 25440, ${ }^{6}$ Natural \\ Product Research Laboratory, School of Pharmacy, Sungkyunkwan University, Suwon 16419, Korea
}

\begin{abstract}
Epigallocatechin gallate (EGCG) and curcumin are well known to naturally-occurring anticancer agents. The aim of this study was to verify the combined beneficial anticancer effects of curcumin and EGCG on PC3 prostate cancer cells, which are resistant to chemotherapy drugs and apoptosis inducers. EGCG showed weaker inhibitory effect on PC3 cell proliferation than two other prostate cancer cell lines, LNCaP and DU145. Co-treatment of curcumin improved antiproliferative effect of EGCG on PC3 cells. The protein expressions of p21 were significantly increased by the co-treatment of EGCG and curcumin, whereas it was not changed by the treatment with each individual compound. Moreover, treatments of EGCG and curcumin arrested both $S$ and G2/M phases of PC3 cells. These results suggest that the enhanced inhibitory effect of EGCG on PC3 cell proliferation by curcumin was mediated by the synergic up-regulation of p21-induced growth arrest and followed cell growth arrest. [BMB Reports 2015; 48(8): 461-466]
\end{abstract}

\section{INTRODUCTION}

Prostate cancer is adenocarcinoma that develops in the prostate gland, a male reproductive organ. Rate of prostate cancer is increasing in the developing as well as developed regions of the world. Factors including age, genetic background and diet have been implicated in the development of prostate cancer

*Corresponding authors. Noriko Yamabe, Tel: +82-31-750-5421; Fax: +82-31-750-5416; E-mail: norikoy@gachon.ac.kr; Ki Sung Kang, Tel: +82-31-750-5402; Fax: +82-31-750-5416; E-mail: kkang@ gachon.ac.kr

${ }^{\#}$ These two authors contributed equally to the work described in this study.

http://dx.doi.org/10.5483/BMBRep.2015.48.8.216

Received 17 October 2014, Revised 6 November 2014, Accepted 1 December 2014

Keywords: Curcumin, Epigallocatechin gallate, Growth arrest, Prostate cancer, p21
(1). Many men with early prostate cancer often have no symptoms or similar symptoms of diseases such as prostatic hyperplasia, and undergo no therapy. Prostate cancer may metastasize from the prostate to other parts of the body, particularly to the bones and lymph nodes (2-4). Globally, prostate cancer-related deaths constitute the sixth leading cause of death in men $(1,5)$.

Various bioactive agents have been used alone or as adjuncts to conventional chemotherapy to improve efficacy and reduce drug-induced toxicity (6). For example, clinical and experimental studies have shown that ingredients in green tea and turmeric roots are beneficial for patients undergoing chemotherapy, by inhibiting proliferation and inducing apoptosis against malignancies, including those of prostate cancer (7-9).

Epigallocatechin gallate (EGCG), also known as eapigallocatechin-3-gallate, is the most abundant catechin in green tea. EGCG has been intensively studied as a chemopreventive agent for head, neck, lung and prostate cancers in vitro and in vivo $(7,8,10,11)$. In prostate cancer, EGCG has been reported to induce changes in intracellular pathways, such as down-regulation of ERK1/2- and Akt-mediated signaling, inhibition of PMA-dependent PKC activation, alteration of Bcl-2 family member ratio and up-regulation of caspases (12-16). Also, inactivation of p53 using small interfering RNA rendered prostate cancer cells resistant to EGCG-mediated apoptosis (17). Curcumin, a component of turmeric, is one of the most commonly used and well-studied phytochemicals. Curcumin has been researched for its anti-tumor effects on various cancer cells and in animal models (18-20). Curcumin inhibits various cell signaling pathways at multiple levels, such as survival, proliferation, enzymes, transcription factors and cell cycle pathways (21).

It is proposed that prevention of cancer is more feasible and holds a better promise with the use of multiple agents that can hit multiple targets in the process of carcinogenesis $(22,23)$. There is also currently an increasing interest in the use of a combination of two or more agents, at low doses with differing modes of action, for chemoprevention (24-27). Consistent with

ISSN: 1976-670X (electronic edition)

Copyright (c) 2015 by the The Korean Society for Biochemistry and Molecular Biology

(c) This is an open-access article distributed under the terms of the Creative Commons Attribution Non-Commercial License (http://creativecommons.org/licenses/by-nc/4.0) which permits unrestricted non-commercial use, distribution, and reproduction in any medium, provided the original work is properly cited. 
this view, we sought to identify whether the combined beneficial anticancer effects of EGCG and curcumin exist in PC3 prostate cancer, with cells that are resistant to many chemotherapy drugs and apoptosis inducers.

\section{RESULTS AND DISCUSSION}

Combinations of natural compounds have been used successfully in the treatment of various types of cancer to gain higher therapeutic effect with lower dosage and to reduce drug resistance and/or side effects (28). Suganuma et al. recently identified that anticancer compounds from edible plants would strengthen their anticancer effect when in combination with green tea catechins (29). In the present study, we have investigated optimal adjuvant candidate of EGCG for prostate cancer treatment and identified its action mechanism.

To compare cell proliferation inhibitory effect of EGCG on three prostate cancer cell types (LNCaP, DU145 and PC3), Ez-cytox cell viability assay was performed at various concentrations for $24 \mathrm{~h}$. As shown in Fig. 1A, prostate cancer cell viability was significantly decreased in a dose-dependent manner by the treatment with EGCG. The $\mathrm{IC}_{50}$ values of EGCG were 159.3, 40.6 and $189.8 \mu \mathrm{M}$ for LNCaP, DU145 and PC3 cells, respectively. Therefore, EGCG showed weaker inhibitory effect on PC3 cell proliferation than in the other prostate cancer cell lines, LNCaP and DU145. This result is in line with earlier studies. In the literature, EGCG showed strong antiproliferative effect and increased the expression of p53 protein and its downstream targets p21 and Bax in prostate cancer $\mathrm{LNCaP}$ cells (28). However, prostate cancer PC3 cell treated with EGCG showed lower anti-proliferation (17).

Next, we compared the anticancer effects of several synthetic and natural anticancer compounds on PC3 cells to determine optimal adjuvant candidate of EGCG for prostate cancer treatment. Two synthetic (cisplatin and etoposide) and two natural compounds (curcumin and ginsenoside $\operatorname{Rg} 3$ ) were chosen based on literature search. As shown in Fig. 1B, all of the synthetic and natural compounds showed anticancer effects on PC3 cells in a dose-dependent manner. The anticancer effects of natural reagents were stronger than synthetic reagents. In particular, the anticancer effect of curcumin on PC3 cells was stronger than other reagents containing EGCG. Therefore, curcumin was suggested to be optimal adjuvant candidate of EGCG.

To evaluate the combined beneficial effect of curcumin on EGCG-induced anticancer action, PC3 cells were treated with EGCG at concentrations of 50 and $100 \mu \mathrm{M}$, in the presence or absence of $50 \mu \mathrm{M}$ curcumin. As shown in Fig. 2A, EGCG inhibited PC3 cell proliferation to 11.2 and $24.3 \%$ at 50 and 100 $\mu \mathrm{M}$, respectively. Curcumin inhibited PC3 cell proliferation to $34.8 \%$ at $50 \mu \mathrm{M}$. In comparison with each treatment of EGCG (50 and $100 \mu \mathrm{M})$ or curcumin $(50 \mu \mathrm{M})$, anticancer effect was markedly improved by the co-treatment of EGCG and curcumin. Also, changes in cellular morphology were ob- served by microscopy as shown in Fig. 2B. A small portion of shrinkage in the cell morphology was shown following curcumin treatment, and progressive condensing and breaking up (karyorrhexis) in the nucleus were shown in most cells after co-treatment of EGCG and curcumin.

Based on the antiproliferative effect of co-treatment of EGCG and curcumin on prostate cancer PC3 cells, its action mechanism was further identified by investigating the expression of tumor survival molecule p-Akt, cell cycle-related protein p21 and $\mathrm{p}-\mathrm{Rb}$ and apoptotic protein caspase 3, using Western blot. As shown in Fig. 3, the expression of tumor survival molecule p-Akt was not changed by the treatments of EGCG, curcumin and co-treatment of EGCG and curcumin. However, the expression level of p21 protein was activated by the treatment of curcumin and more significantly by the co-treatment of EGCG and curcumin. The expression levels of $\mathrm{p}$ - $\mathrm{Rb}$ were slightly decreased by the treatment of EGCG and more strongly by curcumin and co-treatment of EGCG and curcumin. On the other

(A)

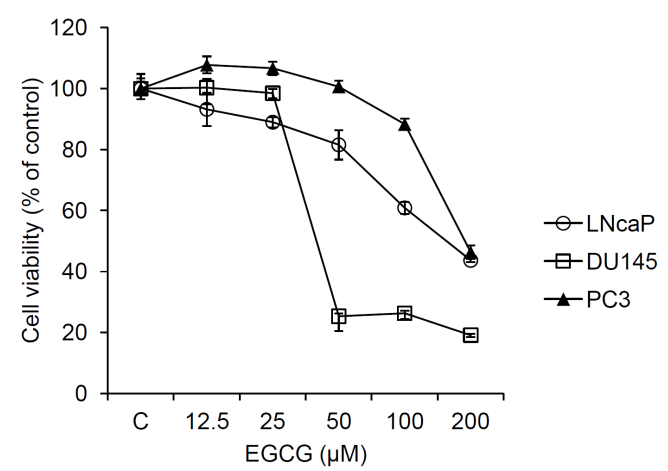

(B)

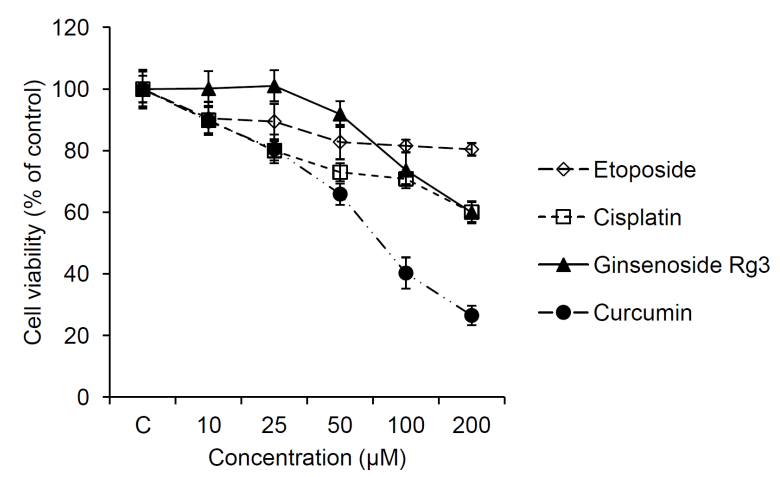

Fig. 1. Inhibitory effects of natural and synthetic anticancer agents on prostate cancer cell proliferation. (A) Comparison in inhibitory effect of EGCG on the proliferation of 3 prostate cancer cells (LNcaP, DU145 and PC3). (B) Comparison in inhibitory effect of etoposide, cisplatin, ginsenoside Rg3 and curcumin on the proliferation of PC3 cells. 
hand, the expression of cleaved caspase 3 was not changed by the treatments of EGCG, curcumin and co-treatment of EGCG and curcumin. Therefore, induction of p21 and inhibition of $\mathrm{p}-\mathrm{Rb}$ may be the main mechanisms related to the inhibition of PC3 cell proliferation.

Cell cycle is one of several anti-cancer targets. Down-regulation of cell cycle on uncontrolled cell proliferation and tumorigenesis can be targeted to suppress cancer progression (30). Either EGCG or curcumin is a strong inhibitor of cell cycle kinases and cell cycle related proteins $(31,32)$. However, the differences in actions of EGCG and curcumin on specific phases of cell cycle are not well known. Co-treatment of the two natural compounds may simultaneously affect multiple cell cycle-related target pathways. To determine whether co-treatment of EGCG and curcumin induced cancer cell cycle

(A)

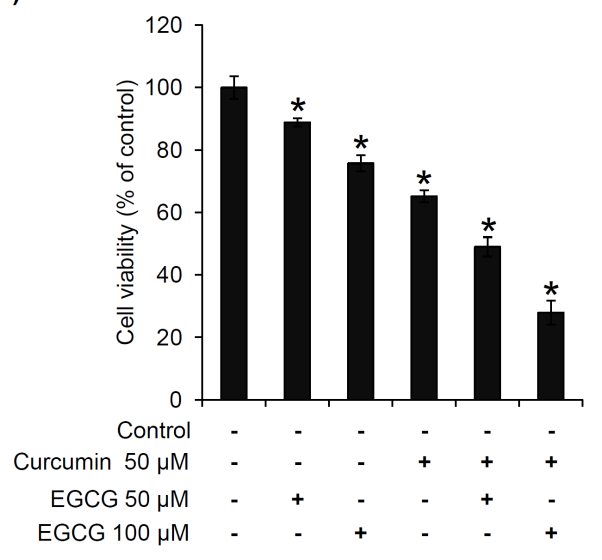

(B)

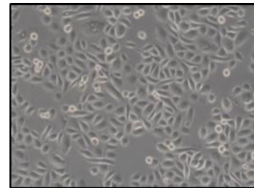

Control

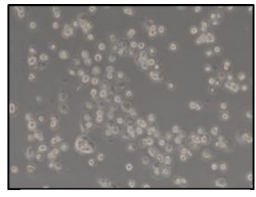

Curcumin $50 \mu \mathrm{M}$

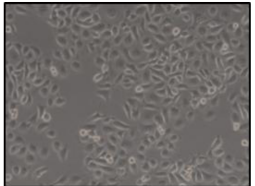

EGCG $100 \mu \mathrm{M}$

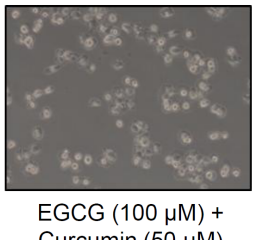

Fig. 2. Combined beneficial effects of EGCG and curcumin on the proliferation of $\mathrm{PC} 3$ prostate cancer cells. (A) Comparison in inhibitory effects of EGCG, curcumin and co-treatment of EGCG and curcumin on the proliferation of PC3 cells. (B) Representative morphological changes in cells confirmed by phase-contrast microscopy. $\mathrm{P}<0.05$ compared to the control value. arrest, flow cytometry analysis was performed to measure the distribution of cell cycles using PI staining. Flow cytometry reveals distribution of cells in three major phases of the cycle (G1 vs. S vs. G2/M) and makes it possible to detect apoptotic cells. As shown in Fig. 4A, EGCG $(100 \mu \mathrm{M})$ mainly arrested S phase of PC3 cells over control cells. However, curcumin (50 $\mu \mathrm{M})$ arrested G2/M phase of PC3 cells over control cells. Arrests in both $\mathrm{S}$ and $\mathrm{G} 2 / \mathrm{M}$ phases of cell cycle were observed by the co-treatment of EGCG and curcumin (Fig. 4B). Therefore, the combined beneficial effect of curcumin on EGCG-induced anticancer effect in PC3 cells was mediated by the induction of their different actions on cell cycle arrest.

While curcumin has versatile anticancer properties, its poor absorption and low bioavailability are the challenges for its developmentas chemopreventive agent (33). The low bioavailability of EGCG is also confirmed in other literature (34). However, it was reported that (-)-epicatechin, another kind of green tea polyphenol, significantly increased the amounts of intracellular curcumin concentration (35), where the exact mechanism is not as yet understood. Further studies on the chemical derivatization or optimal production methods are neededfor clinical application.

In summary, antiproliferative effect of EGCG on PC3 cells was weaker than that on LNCaP and DU145 cells. However, antiproliferative effect of EGCG in PC3 cells was significantly improved after co-treatment of curcumin. The expression levels of $\mathrm{p}-\mathrm{Rb}$ and $\mathrm{p} 21$ were decreased and increased, re-

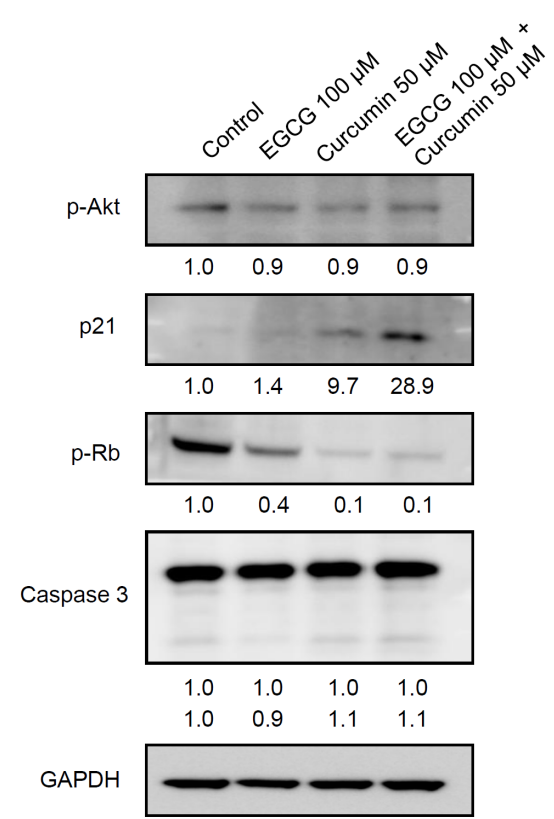

Fig. 3. Combined beneficial effects of EGCG and curcumin on the protein expressions related to apoptosis, cell cycle and tumor suppresser genes in PC3 cells. The band intensities from each blot were quantified using Image J software and normalized to GAPDH. 
(A)

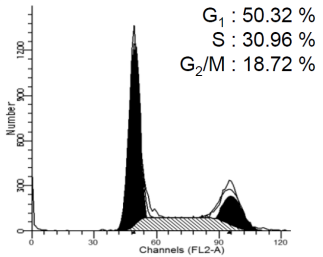

Control

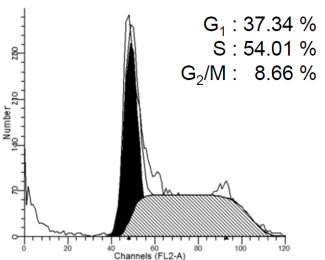

EGCG

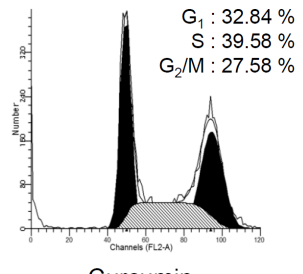

Curcumin

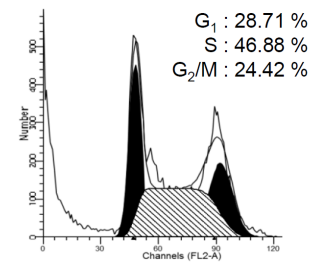

EGCG $(100 \mu \mathrm{M})+$ Curcumin $(50 \mu \mathrm{M})$
(B)

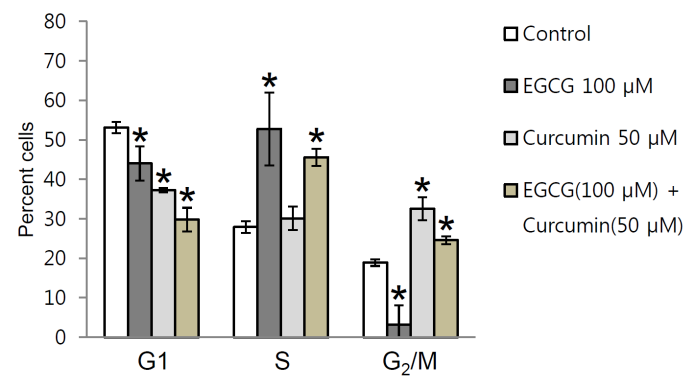

Fig. 4. Combined beneficial effects of EGCG and curcumin on the cell cycle of PC3 cells. (A) Comparison in effects of EGCG, curcumin and co-treatment of EGCG and curcumin on PC3 cell cycle. (B) Quantitative graph for FACS analysis results. $\mathrm{P}<0.05$ compared to the control value.

spectively, after co-treatment of EGCG and curcumin. EGCG and curcumin mainly arrested S and G2/M phases of PC3 cells over control cells, respectively, when treated separately. Co-treatment of EGCG and curcumin arrested both $\mathrm{S}$ and G2/M phases of cell cycle. These results suggest that the enhanced inhibitory effect of EGCG on PC3 cell proliferation by curcumin was mediated by the synergic up-regulation of $\mathrm{p} 21$ and followed cell growth arrest.

\section{MATERIALS AND METHODS}

\section{Chemicals and reagents}

EGCG was purchased from Tocris Bioscience (Bristol, UK). Etoposide, cisplatin, ginsenoside Rg3, curcumin and polyphenon 60 green tea extract were purchased from Sigma Chemical Co. (Missouri, USA). A stock solution of chemicals were prepared in $100 \%$ dimethylsulfoxide (DMSO), and stored at $-20^{\circ} \mathrm{C}$ until use. When required, the stock solution was diluted with cell culture media to the appropriate concentration. The final concentrations of DMSO were adjusted to less than $0.5 \%(\mathrm{v} / \mathrm{v})$ in the culture media.

\section{Cell culture}

The human prostate cancer cells LNCaP, DU145 and PC3 were purchased from the American Type Culture Collection (ATCC, Rockville, USA). LNCaP and PC3 cell were maintained in RPMl-1640 (Gibco, Gaithersburg, USA) medium supplemented with 10\% fetal bovine serum (Gibco, Gaithersburg, USA), 100 units $/ \mathrm{ml}$ penicillin and $100 \mu \mathrm{g} / \mathrm{ml}$ streptomycin and DU145 was grown in Minium Essential Medium (MEM) (Gibco, Gaithersburg, USA) supplemented with $10 \%$ fetal bovine serum, 100 units $/ \mathrm{ml}$ penicillin and $100 \mu \mathrm{g} / \mathrm{ml}$ streptomycin, and incubated at $37^{\circ} \mathrm{C}$ in a humidified incubator with $5 \%$ $\mathrm{CO}_{2}$.

\section{Analysis of cell viability}

Ez-cytox Cell viability assay kit (DOGEN, Seuol, Korea) was used to determine cell viability. Exponentially growing cells were seeded in a 96-well plate at a density of $1 \times 10^{4}$ cells/well in triplicate. The next day, the cells were treated with various samples at various concentrations. After incubation for $24 \mathrm{~h}$, media were changed to avoid interference by the colored compounds. Subsequently, $10 \mu \mathrm{l}$ of the kit reagent was added and the cells were incubated for an additional $1 \mathrm{~h}$. Then, the cell culture supernatant was collected and cell viability was measured by scanning the absorbance of supernatant with a microplate reader at $450 \mathrm{~nm}$. Control cells were exposed to culture media containing $0.5 \%(\mathrm{v} / \mathrm{v})$ DMSO.

\section{Preparation of whole-cell extracts}

Human prostate cancer cells were grown in $60 \mathrm{~mm}$ dishes and treated with various concentration of EGCG, curcumin and mixture of $100 \mu \mathrm{M}$ EGCG and $50 \mu \mathrm{M}$ curcumin or were not treated for $24 \mathrm{~h}$. Whole cell extracts were prepared according to the manufacturer's instructions using RIPA buffer (Cell Signaling, Beverly, USA) supplemented with $1 x$ protease inhibitor cocktail and $1 \mathrm{mM}$ phenylmethylsulfonyl fluoride (PMSF).

\section{Westem blot analysis}

Proteins (whole cell extracts: $30 \mu \mathrm{g} /$ lane) were separated by electrophoresis through a NuPAGE 4-12\% Bis-tris gel (Invitrogen, Carlsbad, USA), blotted onto a PVDF transfer membrane, and analyzed with the epitope-specific primary and secondary antibodies. The bound antibodies were visualized using ECL Advance Western Blotting Detection Reagents (GE Healthcare, Waukesha, USA) and LAS-4000 (Fujifilm, Tokyo, Japan). Monoclonal antibodies to p-Akt (Ser 473), p21, GAPDH and ployclonal antibodies to PTEN, Akt, p53, Bcl-2 and p-Rb were purchased from Cell Signaling Technology, Inc. (Beverly, USA). The band intensities from each blot were quantified using Image J software and normalized to GAPDH. 


\section{Flow cytometric analysis}

Cell cycle arrest was assessed by PI staining using an Annexin-V-FLUOS staining Kit (Roche, Indianapolis, USA) according to the manufacturer's recommendations. Briefly, whole cells were collected, rinsed with DPBS, incubated with $300 \mu$ of DPBS involved $10 \mu \mathrm{g} / \mathrm{ml}$ RNase A for $15 \mathrm{~min}$ and $12 \mu \mathrm{l}$ of PI at RT for 15 min in the dark, and analyzed by a FACSCalibur flow cytometer. At least 20000 events were evaluated in these experiments.

\section{Statistical analysis}

Statistical significance was determined through one-way analysis of variance (ANOVA) followed by a multiple comparison test with a Bonferroni adjustment. P values of less than 0.05 were considered statistically significant.

\section{ACKNOWLEDGEMENTS}

This research was funded by Gangneung Asan Hospital Biomedical Research Center Promotion Fund. This research was also carried out with the support of the Korea Institute of Science and Technology institutional program (2Z04230). This research was conducted under the industrial infrastructure program for fundamental technologies (N0000885) which is funded by the Ministry of Trade, Industry \& Energy (MOTIE, Korea). This article is a revision of the first author's master's thesis from Gangneung-Wonju National University.

\section{REFERENCES}

1. Siegel R, Ward E, Brawley O and Jemal A (2011) Cancer statistics, 2011: the impact of eliminating socioeconomic and racial disparities on premature cancer deaths. CA Cancer J Clin 61, 212-236

2. Lange PH and Vessella RL (1998) Mechanisms, hypotheses and questions regarding prostate cancer micrometastases to bone. Cancer Metastasis Rev 17, 331-336

3. Bubendorf L, Schopfer A, Wagner U et al (2000) Metastatic patterns of prostate cancer: An autopsy study of 1,589 patients. Hum Pathol 31, 578-583

4. Roudier MP, Morrissey C, True LD, Higano CS, Vessella RL and Ott SM (2008) Histopathological assessment of prostate cancer bone osteoblastic metastases. J Urol 180, 1154-1160

5. Ferlay J, Shin HR, Bray F, Forman D, Mathers C and Parkin DM (2010) Estimates of worldwide burden of cancer in 2008: GLOBOCAN 2008. Int J Cancer 127, 2893-2917

6. Vickers A (2002) Botanical medicines for the treatment of cancer: rationale, overview of current data, and methodological considerations for phase I and II trials. Cancer Invest 20, 1069-1079

7. Shimizu M, Adachi S, Masuda M, Kozawa O and Moriwaki H (2011) Cancer chemoprevention with green tea catechins by targeting receptor tyrosine kinases. Mol Nutr Food Res 55, 832-843

8. Yang H, Landis-Piwowar K, Chan TH and Dou QP (2011)
Green tea polyphenols as proteasome inhibitors: Implication in chemoprevention. Cancer Drug Targets 11, 296306

9. Sintara K, Thong-Ngam D, Patumraj S and Klaikeaw N (2012) Curcumin attenuates gastric cancer induced by $\mathrm{N}$-methyl-N-nitrosourea and saturated sodium chloride in rats. J Biomed Biotechnol 2012, 1-8

10. Yang CS, Wang X, Lu G and Picinich SC (2009) Cancer prevention by tea: Animal studies, molecular mechanisms and human relevance. Nat Rev Cancer 9, 429-439

11. Kim JW, Amin AR and Shin DM (2010) Chemoprevention of head and neck cancer with green tea polyphenols. Cancer Prev Res 3, 900-909

12. Ahmad N, Gupta S and Mukhtar H (2000) Green tea polyphenol epigallocatechin-3-gallate differentially modulates nuclear factor kappaB in cancer cells versus normal cells. Arch Biochem Biophys 376, 338-346

13. Adhami VM, Ahmad N and Mukhtar H (2003) Molecular targets for green tea in prostate cancer prevention. J Nutr $133,2417-2424$

14. Borska S, Gebarowska E, Wysocka T, Drag-Zalesinska M and Zabel M (2003) Induction of apoptosis by EGCG in selected tumour cell lines in vitro. Folia Histochem Cytobiol 41, 229-232

15. Ahmad KA, Harris $\mathrm{NH}$, Johnson AD, Lindvall HC, Wang G and Ahmed K (2007) Protein kinase CK2 modulates apoptosis induced by resveratrol and epigallocatechin3-gallate in prostate cancer cells. Mol Cancer Ther 6, 1006-1012

16. Chung LY, Cheung TC, Kong SK et al (2001) Induction of apoptosis by green tea catechins in human prostate cancer DU145 cells. Life Sci 68, 1207-1214

17. Hastak K, Agarwal MK, Mukhtar $\mathrm{H}$ and Agarwal ML (2005) Ablation of either p21 or Bax prevents p53-dependent apoptosis induced by green tea polyphenol epigallocatechin-3-gallate. FASEB J 19, 789-791

18. Kuttan G, Kumar KB, Guruvayoorappan $C$ and Kuttan $R$ (2007) Antitumor, anti-invasion, and antimetastatic effects of curcumin. Adv Exp Med Biol 595, 173-184

19. Kunnumakkara AB, Anand P and Aggarwal BB (2008) Curcumin inhibits proliferation, invasion, angiogenesis and metastasis of different cancers through interaction with multiple cell signaling proteins. Cancer Lett 269, 199-225

20. Zhang X, Chen LX, Ouyang L, Cheng Y and Liu B (2012) Plant natural compounds: Targeting pathways of autophagy as anti-cancer therapeutic agents. Cell Prolif 45, 466-476

21. Shehzad A, Wahid F and Lee YS (2010) Curcumin in cancer chemoprevention: Molecular targets, pharmacokinetics, bioavailability, and clinical trials. Arch Pharm 343, 489-499

22. Mukhtar H and Ahmad N (1999) Cancer chemoprevention: future holds in multiple agents. Toxicol Appl Pharmacol $158,207-210$

23. McCarty MF (2004) Targeting multiple signaling pathways as a strategy for managing prostate cancer: multifocal signal modulation therapy. Integr Cancer Ther 3, 349-380

24. Banerjee S, Zhang Y, Ali S et al (2005) Molecular evidence for increased antitumor activity of gemcitabine by 
genistein in vitro and in vivo using an orthotopic model of pancreatic cancer. Cancer Res 65, 9064-9072

25. Khor TO, Keum YS, Lin W et al (2006) Combined inhibitory effects of curcumin and phenethyl isothiocyanate on the growth of human PC-3 prostate xenografts in immunodeficient mice. Cancer Res 66, 613-621

26. Narayanan BA, Narayanan NK, Pttman B and Reddy BS (2006) Adenocarcina of the mouse prostate growth inhibition by celecoxib: downregulation of transcription factors involved in COX-2 inhibition. Prostate 66, 257-265

27. Adhami VM, Malik A, Zaman N et al (2007) Combined inhibitory effects of green tea polyphenols and selective cyclooxygenase-2 inhibitors on the growth of human prostate cancer cells both in vitro and in vivo. Clin Cancer Res $13,1611-1619$

28. Chou TC (2006) Theoretical basis, experimental design, and computerized simulation of synergism and antagonism in drug combination studies. Pharmacol Rev 58, 621-681

29. Suganuma M, Saha A and Fujiki H (2011) New cancer treatment strategy using combination of green tea catechins and anticancer drugs. Cancer Sci 102, 317-323
30. Stone A, Sutherland RL and Musgrove EA (2012) Inhibitors of cell cycle kinases: Recent advances and future prospects as cancer therapeutics. Crit Rev 17, 175-198

31. Chauhan DP (2002) Chemotherapeutic potential of curcumin for colorectal cancer. Curr Pharm 8, 1695-1706

32. Shankar S, Suthakar G and Srivastava RK (2007) Epigallocatechin-3-gallate inhibits cell cycle and induces apoptosis in pancreatic cancer. Front Biosci 12, 5039-5051

33. Saha A, Kuzuhara T, Echigo N, Suganuma M and Fujiki H (2010) New role of (-)-epicatechin in enhancing the induction of growth inhibition and apoptosis in human lung cancer cells by curcumin. Cancer Prev Res 3, 953-962

34. Kim S, Lee MJ, Hong J et al (2000) Plasma and tissue levels of tea catechins in rats and mice during chronic consumption of green tea polyphenols. Nutr Cancer 37, 41-48

35. Somers-Edgar TJ, Scandlyn MJ, Stuart EC, le Nedelec MJ, Valentine SP and Rosengren RJ (2008) The combination of epigallocatechin gallate and curcumin suppresses ER alpha-breast cancer cell growth in vitro and in vivo. Int J Cancer 122, 1966-1971 